\title{
Análise ultrassônica de meios ortorrômbicos sintéticos
}

\author{
João Rafael B. Silveira* (Universidade Federal do Pará, Brasil), Léo Kirchhof Santos (Universidade Federal do Pará, Brasil), \\ José J. S. de Figueiredo (Universidade Federal do Pará, Brasil \& INCT-GP, Brasil) e Lucas C. Costa(Universidade Federal do \\ Pará, Brasil)
}

\begin{abstract}
Copyright 2018, SBGf - Sociedade Brasileira de Geofísica
This paper was prepared for presentation during the $8^{\text {th }}$ Brazilian Symposium on Geophysics, held in Salinópolis, Brazil, 18 to 20 setember 2018 .

Contents of this paper were reviewed by the Technical Committee of the $8^{\text {th }}$ Brazilian Symposium on Geophysics and do not necessarily represent any position of the SBGf, its officers or members. Electronic reproduction or storage of any part of this paper for commercial purposes without the written consent of The Brazilian Geophysical Society is prohibited.
\end{abstract}

\section{Abstract}

The study of fractures in subsurface is very important since they are, in some cases, the main conduits for hydrocarbon flow in a reservoir. There are many ways to study the behavior of seismic waves for different rock properties, including the use of physical modeling. This procedure is based on the performing of ultrasonic measurements in reduced-scale rocks and is used to analyze the behavior of seismic waves in different geologic cases, as well as for the calibration of effective medium theories. In this work, by performing ultrasonic measurements, we estimated Pwave velocities on a group of samples, including ones with orthorhombic symmetry. In the orthorhombic samples it was shown a great influence of fractures on the waves propagating through them, with the graphs showing an almost quadratic behavior. On the other hand, the velocities of waves propagating through the layering and fracture planes have their magnitude almost linear for VTI and orthorhombic samples. It is great to highlight that the construction methodology used in this work was successful in reproducing complex media, as the orthorhombic samples.

\section{Introdução}

Fraturas são descontinuidades existentes em rochas em subsuperfície e são criadas quando a tensão aplicada a uma rocha é maior que sua resistência. Elas são amplamente distribuídas no interior da Terra, tendo uma grande variedade de tamanhos e orientações. Em alguns reservatórios, os chamados reservatórios fraturados, elas atuam como o principal conduto para o fluxo de hidrocarbonetos. Portanto, é de vital importância o estudo e a caracterização de fraturas em subsuperfície com o objetivo de otimizar a produção de hidrocarbonetos em um reservatório (Nelson, 1985; Sayers, 2007). Uma maneira de investigar, qualificar e quantificar as propriedades de feições geológicas em subsuperfície, incluindo zonas fraturadas, é a partir da análise do comportamento das propriedades de ondas sísmicas (velocidade, amplitude, dispersão,etc.) ao se propagarem por diferentes meios (Crampin, 1981; Crampin et al., 1980).

Há varias maneiras de se analisar o comportamento de ondas sísmicas em diferentes situações geológicas. Uma maneira é a partir da modelagem numérica, processo no qual se modela uma resposta sísmica a partir da propagação de ondas com a utilização de algum método numérico ou a partir de traçamento de raios. Essa análise também pode ser feita a partir da utilização das chamadas teorias de meios efetivos (Hudson, 1980, 1981; Hudson and Liu, 1999), que são equações que expressam mudanças nas propriedades das ondas sísmicas em termos das propriedades do meio pelo qual elas se propagam. No estudo de meios fraturados, elas expressam, por exemplo, como as velocidades de ondas $\mathrm{P}$ e $\mathrm{S}$ variam em função da forma das fraturas, densidade de fratura e fluido saturante. Outra maneira de se estudar o comportamento das propriedades de ondas sísmicas em diferentes situações geológicas é através do processo de modelagem física. Esse processo consiste na reprodução de rochas em escala reduzida, com parâmetros geológicos controlados. Muitos estudos utilizam a modelagem física para realizar uma grande variedade de abordagens, como a análise da variação das velocidades de ondas $\mathrm{P}$ e $\mathrm{S}$ para diferentes densidades de fratura ou fluido saturante (Ding et al., 2014; Ass'ad et al., 1992; Tillotson et al., 2012; de Figueiredo et al., 2012) ou mesmo o estudo da atenuação sofrida pelas ondas em função de diferentes propriedades (Amalokwu et al., 2014). Os estudos envolvendo modelos físicos são, em geral, realizados em laboratório, com auxílio de fontes de ondas sísmica de altíssima frequência, os chamados transdutores ultrassônicos. Além da análise do comportamento da onda sísmica para diferentes características geológicas, o processo de modelagem física pode ser também utilizado para calibrar teorias de meios efetivos (Ass'ad et al., 1996; Rathore et al., 1995).

O objetivo do trabalho é caracterizar o comportamento da onda compressional $(P)$ ao se propagar em rochas sintéticas representando arenitos com simetrias isotrópica, verticalmente transversalmente isotrópica (VTI) e ortorrômbica, e comparar os resultados encontrados para cada um dos casos. A simetria ortorrômbica, em termos geológicos, pode ser representado por um conjunto de fraturas verticais existentes em um meio com acamadamento. Já o meio VTI pode ser representado por um meio com acamadamento. Trabalhos experimentais realizados com meios ortorrômbicos são raramente feitos devido a complexidade de sua construção, o que aumenta mais ainda a importância do presente trabalho. As medidas ultrassônicas são realizadas com transdutores de frequência igual a $1 \mathrm{MHz}$, a partir do procedimento de transmissão de ondas, semelhante ao realizado em Santos et al. $(2015,2016)$. 


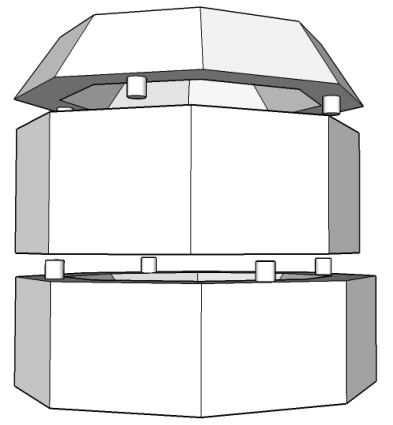

Figura 1: Projeto dos modelos das fôrmas.

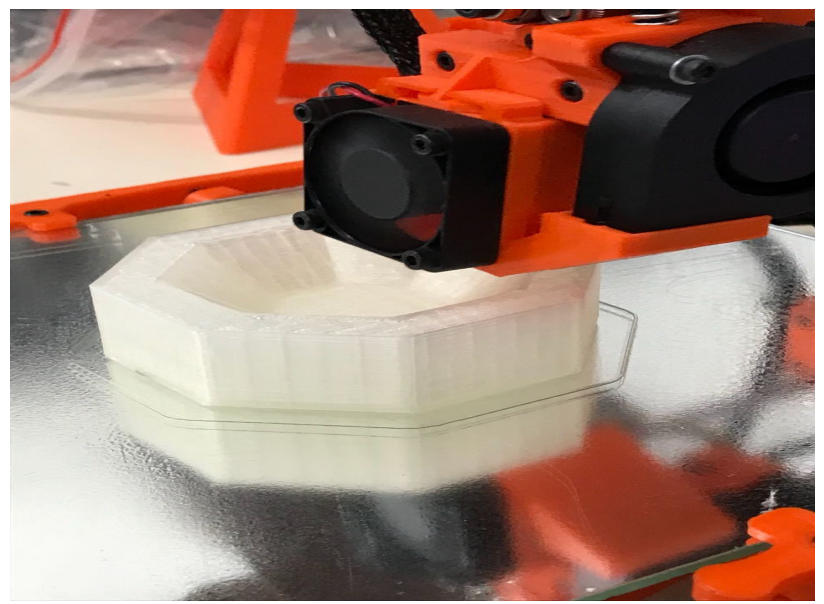

Figura 2: Impressora 3D em funcionamento durante a confecção dos moldes que darão o formato às amostras.

\section{Construção das rochas sintéticas}

A base para a construção das amostras é uma mistura de $70 \%$ de areia e $30 \%$ de cimento que, ao serem misturados a água, dão origem ao material que origina a matriz isotrópica. Esta mistura é, então, despejada no interior dos moldes que darão forma às rochas sintéticas. No caso da amostra isotrópica, a mistura é despejada de uma única vez no interior do molde. Para o caso da amostra VTI e das amostras ortorrômbicas, a mistura é despejada de modo a formar camadas com $5 \mathrm{~mm}$ de espessura, tendo uma diferença de vinte minutos de deposição para cada camada. As fraturas, existentes apenas nas amostras ortorrômbicas, são originadas a partir da dissolução de tiras quadradas de isopor, de espessura igual a $1 \mathrm{~mm}$, que são posicionadas perpendicularmente aos planos de acamadamento ver Figura 2. A dissolução das tiras de isopor é realizada por Thinner e tem como resultado a criação de espaços vazios no interior das amostras. Os moldes que dão forma às amostras foram projetados usando o programa SketchUp e impressos usando uma impressora $3 \mathrm{D}$ ver Figura 1, tendo como matéria prima filamentos de Polyactic Acid (PLA). Esta metodologia de construção foi desenvolvida por Santos et al. (2017) e está melhor detalhada no mesmo.

Ao todo foram construídas cinco amostras, sendo uma

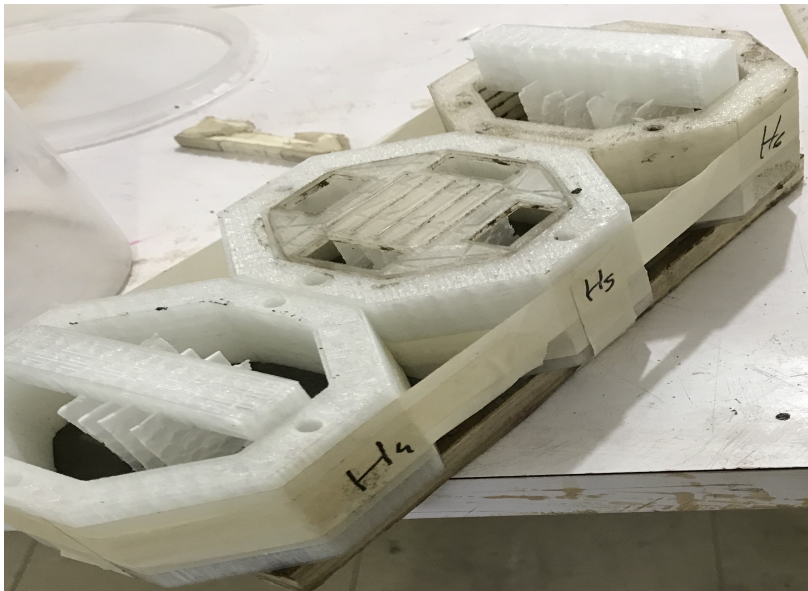

Figura 3: Tiras quadradas de isopor dispostas no interior das amostras durante o processo de construção. Elas dão origem aos espaços vazios representativos de fraturas.

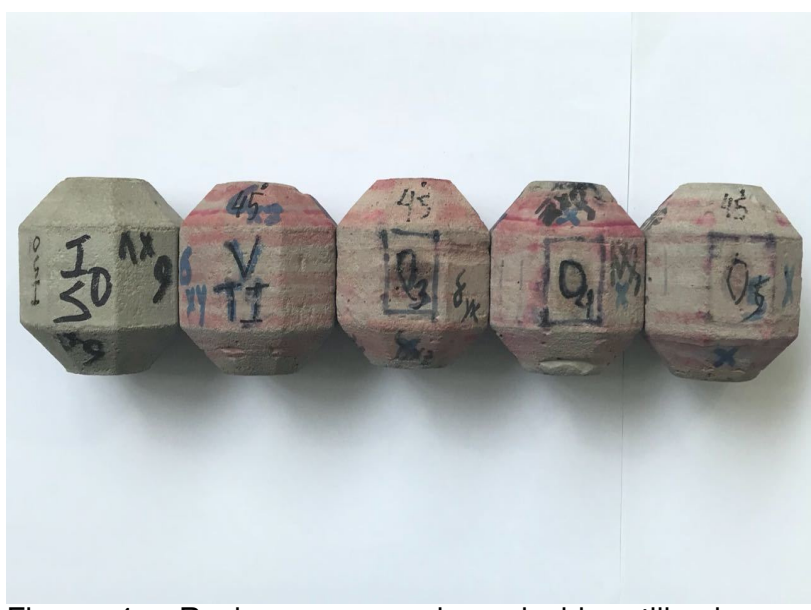

Figura 4: Rochas em escala reduzida utilizadas no trabalho. É possível ver que suas formas possibilitam aquisição de ondas $\mathrm{P}$ diagonalmente.

isotrópica, uma com simetria VTI e três amostras ortorrômbicas. As amostras ortorrômbicas se diferenciam entre si pelo número de fraturas existentes em seu interior, havendo amostras que possuem três (Amostra O3), quatro (Amostra O4) e cinco (Amostra O5) fraturas. As amostras construidas possuem um formato que permite a aquisição de sinais ultrassônicos diagonalmente ao conjunto de fissuras ver Figura 4).

\section{Medidas ultrassônicas}

As medidas ultrassônicas foram realizadas no Laboratório de Petrofísica e Física de Rochas Dr. Om Prakash Verma, localizado na Universidade Federal do Pará. Elas se basearam na técnica de transmissão de pulso (Santos et al., 2015, 2016) e foram realizadas com a utilização do Sistema de Aquisição Ultrassônico existente no laboratório. O sistema consiste em: um pulse-receiver 5072PR e um pré-amplificador 5660B da Olympus; um par de transdutores ultrassônicos de onda $\mathrm{P}$, de $1 \mathrm{MHz}$; e um osciloscópio USB de $50 \mathrm{MHz}$ da Handscope. A partir do tempo de propagação da onda em uma cada direção de 
propagação, estima-se a velocidade a partir da seguinte equação:

$$
V=\frac{L}{t-t_{\text {delay }}},
$$

onde L é a distância percorrida pela onda, t é o seu tempo de primeira chegada e $t_{\text {delay }}$ é o atraso intrínseco existentes no transdutores que, neste caso, é igual a $0.14 \mu \mathrm{s}$. A taxa de amostragem para cada canal em todas as medidas foi de $0.1 \mu \mathrm{s}$

Para todas as amostras, as velocidades foram estimadas nas direções perpendicular ao plano das fraturas (direção $X$ ), paralela ao plano das fraturas e ao plano de acamadamento (direção $Y$ ) e paralela ao plano das fraturas e perpendicular ao plano de acamadamento (direção Z). Além dessas três direções, houve estimativa de velocidades em direções diagonal ao acamadamento (plano YZ), diagonal ao plano de fraturas e paralelo ao acamadamento (Direção $X Y$ ) e diagonal ao plano de fraturas e ao acamadamento (plano $X Z$ ). As direções de transmissão a partir das quais foram estimadas as velocidades de onda $P$ estão detalhadas nas Figuras $6 a$ e $6 b$.

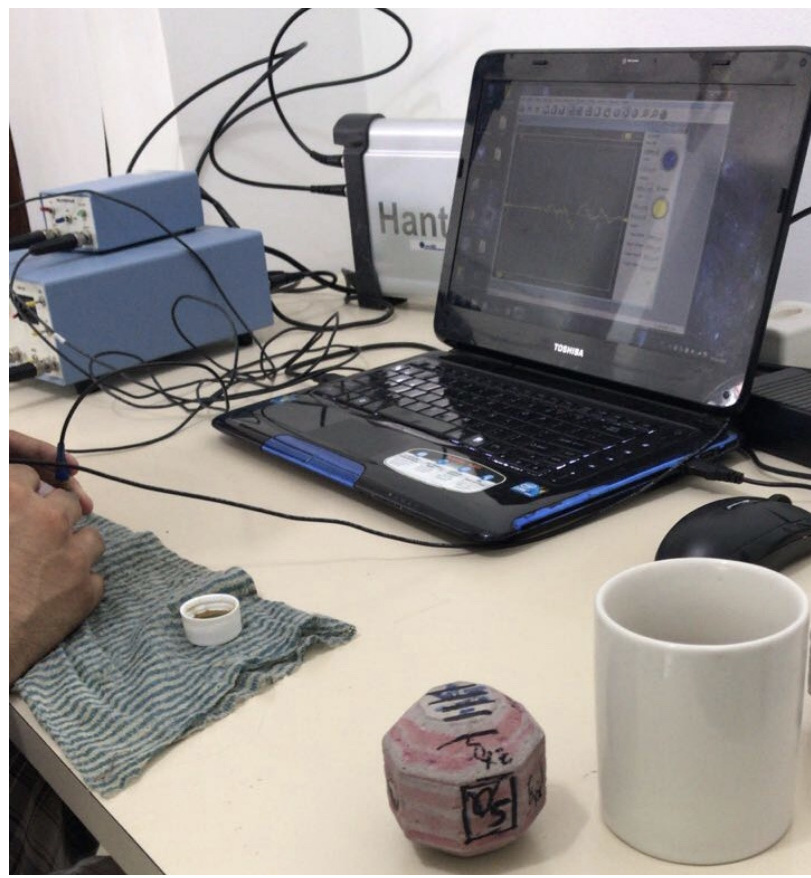

Figura 5: Medidas ultrassônicas sendo realizadas utilizando os transdutores.

\section{Resultados e Discussões}

Os resultados são mostrados nas Figuras 7a e 7b e representam, respectivamente, a variação das velocidades de onda $P$ nas direções $X, Y$ e $Z$ e a variação das velocidades nas direções diagonais $X Y$, $X Z$ e $Y Z$ para cada uma das amostras construídas. Começando a interpretação pela Figura $7 a$, é visível que as velocidades têm valores muito próximos entre si para a amostra isotrópica. Esse valor é condizente com a teoria, já que um meio isotrópico é considerado aquele em que não há variação de velocidade independente da direção de propagação da onda. A velocidade da onda que se
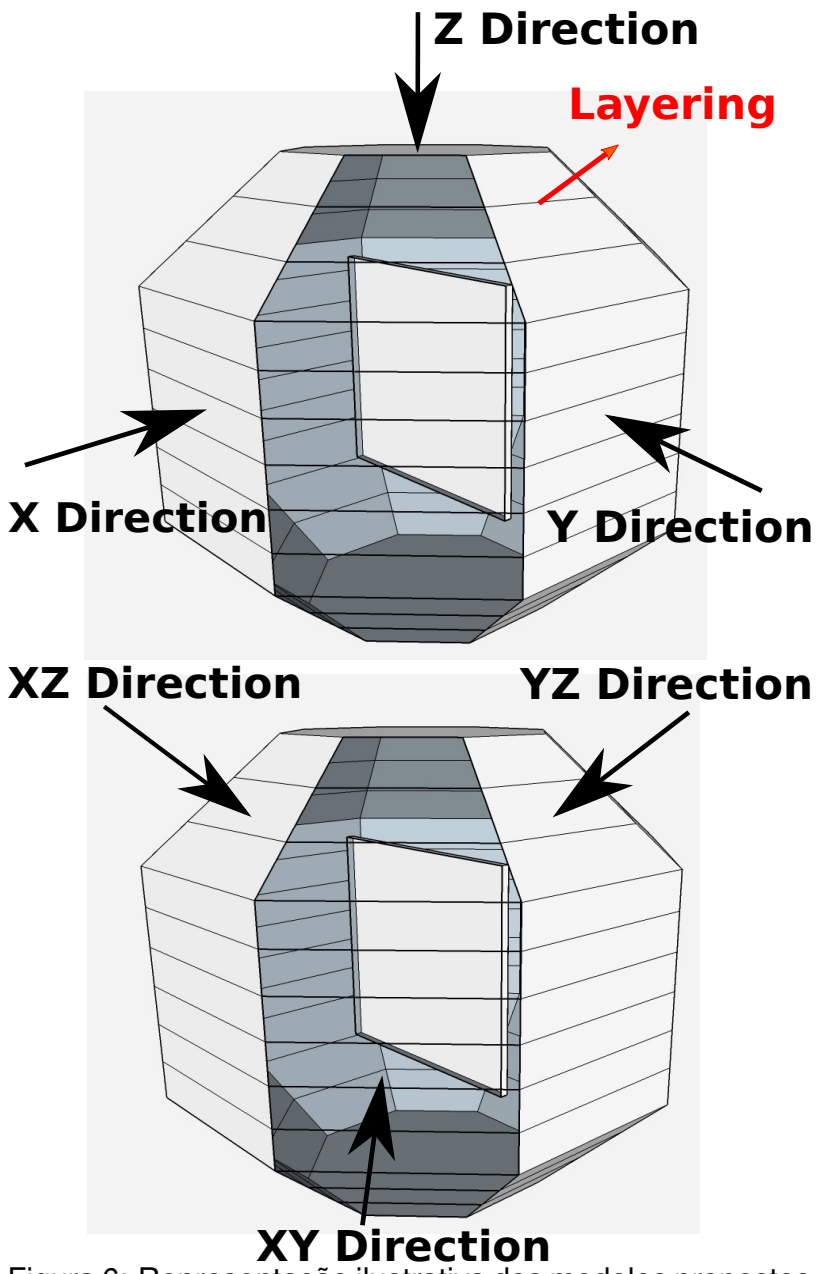

Figura 6: Representação ilustrativa dos modelos propostos por Hudson and Liu (1999).

propaga em $\mathrm{Y}$ tem uma variação muito pequena para as amostras, tendo um comportamento quase constante. Isso ocorre porque, nessa direção, a onda não ultrapassa nenhum "obstáculo", ou seja, não há transmissão da onda por fraturas ou interfaces, diminuindo a dispersão. A velocidade da onda que se propaga na direção $Z$ possui um comportamento também aproximadamente constante para a amostra VTI e para as fraturadas, porém, com magnitude bem inferiores ao caso de $\mathrm{V}_{P y}$. $\mathrm{A}$ uniformidade nas velocidades é explicada pelo fato de o acamadamento nas amostras VTI e ortorrômbicas ser o mesmo, inclusive com o mesmo número de camadas. A queda do valor de velocidade em relação aos valores $\mathrm{V}_{P y}$ ocorre pelo fato de as ondas, quando se propagam na direção $Z$, encontram as interfaces entre cada camada, o que configura um obstáculo e acarreta em perda de energia e velocidade da onda. Diferentemente dos dois casos anteriores, a velocidade da onda que se propaga na direção $X$ apresenta uma visível variação de acordo com o aumento do número de fraturas existentes nas amostras, apresentando um comportamento descrescente, de tendência aproximadamente quadrática. Esse comportamento evidencia a grande influência que as fraturas causam nas velocidades de onda $P$, diminuindo significantemente sua velocidade. Agora observando a Figura7b, os menores valores observados 
a)

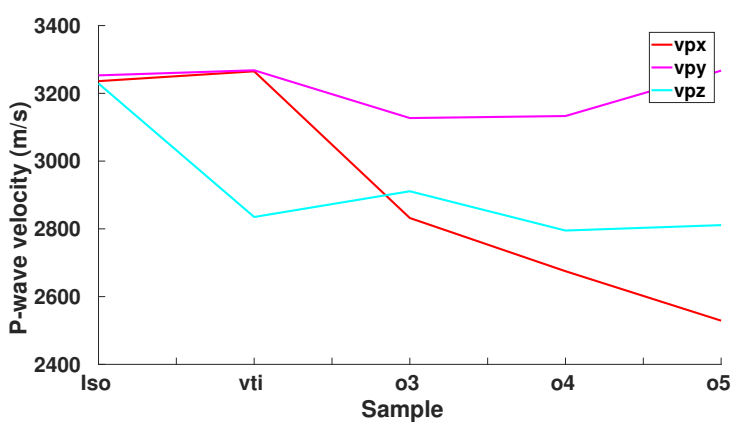

b)

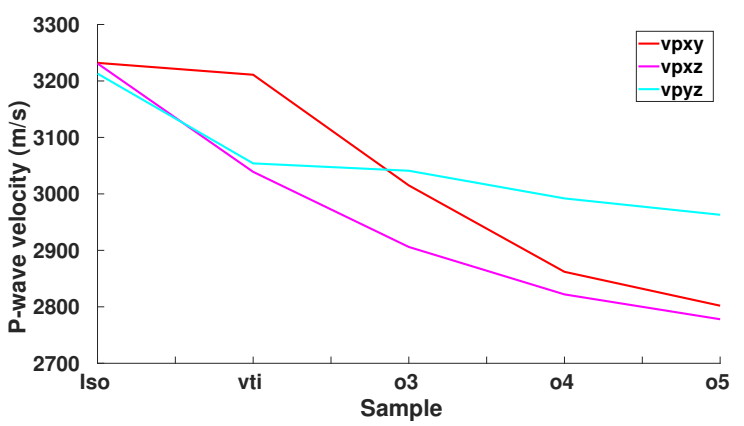

Figura 7: Variação das velocidades de onda $P$ em função das direções a) $x$, y e $z$ e b) dos planos xy, xz e yz nas amostras analisadas

são em $\mathrm{V}_{P x z}$, tem valores decrescentes nas amostras ISO para VTI,e diminuindo continuamente com o aumento de descontinuidades, pois a onda passa tanto pelo acamadamento horizontal como pelas fraturas, assim tendo a maior perda de energia e consequentemente os menores valores, já $\mathrm{V}_{P y z}$, sendo maior na Iso, sem acamadamento, menor e constante nas amostras VTI, O3, O4 E O5 apresentando continuidade independente do número de fraturas, pois a onda esta percorrendo através do acamadamento horizontal, já $\mathrm{V}_{P x y}$ constante em iso e vti e decrescentes de acordo com o aumento de fraturas, só tem a influência das mesmas, todas amostras observadas estão seguindo um comportamento previsto teoricamente.

\section{Conclusões}

Foi evidenciado que as ondas $\mathrm{P}$ e $\mathrm{S}$ se propagando no plano das fraturas (direção $Y$ ) e perpendicular ao acamadamento (direção $Z$ ) possuem o mesmo comportamento tanto para as amostras ortorrômbicas quanto para a VTI, o que corrobora com a teoria descrita para meios desse tipo (Tsvankin, 1997; Hudson, 1981). Por sua vez, a velocidade $\mathrm{V}_{P x}$ mostrou-se extremamente sensível a presença do número de fraturas, tendo um comportamento linear, além disso a velocidade $\mathrm{V}_{P x z}$ e $\mathrm{V}_{P x y}$ apresentam um comportamento quadrático, ou seja, não linear, com o aumento do número de fraturas.

A metodologia de construção proposta por Santos et al. (2017) mostrou-se, além de prática, aplicável a situações mais complexas, como o caso da construção de rochas com simetria ortorrômbica. Isso foi constatado pelo

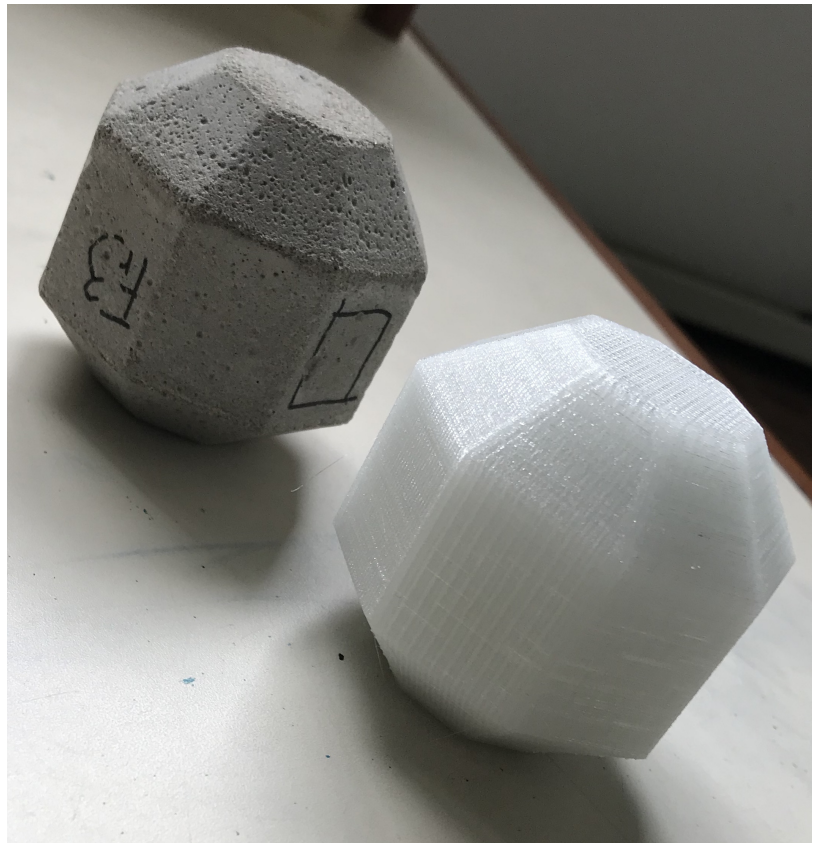

Figura 8: comparação entre uma amostra utilizando os parâmetros desse artigo com uma amostra inteiramente criada usando a impressora 3D.

comportamento das velocidades estimadas neste trabalho assim condizentes com o previsto para rochas desse tipo.

Com o intuito de dar continuidade ao presente trabalho, a ideia é criar dois conjuntos diferentes de amostras com simetrias $\mathrm{VTI}$, transversalmente isotrópico com eixo de simetria da horizontal (HTI) e ortorrômbica. Um dos conjuntos será produzido utilizando a metodologia de construção mostrada neste trabalho, enquanto o outro será produzido em uma impressora 3D. Alguns testes já realizados em nosso laboratório mostraram que é possível criar modelos com as mesmas propriedades físicas dos utilizados neste trabalho a partir de uma impressora, utilizando como matéria prima um plástico chamado PLA (Figura 8). O intuito com os dois conjuntos de amostras é demonstrar, de forma experimental, que meios com simetria ortorrômbica podem ser representados como a soma de meios com simetrias VTI e HTI. Além disso, a ideia é realizar o cálculo dos parâmetros de Thomsen, incluindo o parâmetro $\delta$, para todos as amostras.

\section{Referências}

Amalokwu, K., A. I. Best, J. Sothcott, M. Chapman, T. Minshull, and X.-Y. Li, 2014, Water saturation effects on elastic wave attenuation in porous rocks with aligned fractures: Geophysical Journal International, 197, 943947.

Ass'ad, J. M., J. A. Mcdonald, R. H. Tatham, and T. M. Kusky, 1996, Elastic Wave Propagation In A Medium Containing Oriented Inclusions With A Changing Aspect Ratio: A Physical Model Study: Geophysical Journal International, 125, 163-172.

Ass'ad, J., R. Tatham, and J. McDonald, 1992, A physical model study of microcrackinduced anisotropy: GEOPHYSICS, 57, 1562-1570.

Crampin, S., 1981, A review of wave motion in anisotropic and cracked elastic-media: Wave Motion, 3, 343-391. 
Crampin, S., R. Evans, B. Üçer, M. Doyle, J. P. Davis, G. V. Yegorkina, and A. Miller, 1980, Observations of dilatancy-induced polarization anomalies and earthquake prediction: Nature, 286, 874.

de Figueiredo, J. J. S., J. Schleicher, R. R. Stewart, and N. Dyaur, 2012, Estimating fracture orientation from elasticwave propagation: An ultrasonic experimental approach: Journal of Geophysical Research: Solid Earth, 117, B08304.

Ding, P., B. Di, J. Wei, X. Li, and Y. Deng, 2014, Fluiddependent anisotropy and experimental measurements in synthetic porous rocks with controlled fracture parameters: Journal of Geophysics and Engineering, 11, 015002.

Hudson, J., and E. Liu, 1999, Effective elastic properties of heavily faulted structures: GEOPHYSICS, 64, 479-485.

Hudson, J. A., 1980, Overall properties of a cracked solid: Mathematical Proceedings of the Cambridge Philosophical Society, 88, 371-384.

, 1981, Wave speeds and attenuation of elastic waves in material containing cracks: Geophysical Journal of the Royal Astronomical Society, 64, 133-150.

Nelson, R. A., 1985, Geologic analysis of naturally fractured reservoirs: Gulf Pub. Co., Book Division.

Rathore, J., E. Fjaer, R. Holt, and L. Renlie, 1995, P- and Swave anisotropy of a synthetic sandstone with controlled crack geometryl1: Geophysical Prospecting, 43, 711728.

Santos, L. K., J. J. S. de Figueiredo, and C. B. da Silva, 2016, A study of ultrasonic physical modeling of isotropic media based on dynamic similitude: Ultrasonics, $\mathbf{7 0}$, 227-237.

Santos, L. K., J. J. S. de Figueiredo, B. Omoboya, J. Schleicher, R. R. Stewart, and N. Dyaur, 2015, On the source-frequency dependence of fracture-orientation estimates from shear-wave transmission experiments: Journal of Applied Geophysics, 114, 81-100.

Santos, L. K., J. J. S. de Figueiredo, C. B. Silva, D. L. Macedo, and A. M. Leandro, 2017, A new way to construct synthetic porous fractured medium: Journal of Petroleum Science and Eng.

Sayers, C., 2007, Seismic characterization of reservoirs containing multiple fracture sets, in SEG Technical Program Expanded Abstracts 2007: Society of Exploration Geophysicists, SEG Technical Program Expanded Abstracts, 1456-1460.

Tillotson, P., J. Sothcott, A. I. Best, M. Chapman, and X.-Y. $\mathrm{Li}, 2012$, Experimental verification of the fracture density and shear-wave splitting relationship using synthetic silica cemented sandstones with a controlled fracture geometry: Geophysical Prospecting, 60, 516-525.

Tsvankin, I., 1997, Anisotropic parameters and Pwave velocity for orthorhombic media: GEOPHYSICS, 62, 1292-1309. 